\title{
Design, fabrication and testing of elliptical crystal bender for the EXAFS beam-line at INDUS-II synchrotron source
}

\author{
N C DAS, S N JHA, D BHATTACHARYYA*, A K POSWAL \\ $\mathrm{A} \mathrm{K} \mathrm{SINHA}^{+}$and $\mathrm{V} \mathrm{K} \mathrm{MISHRA}{ }^{+}$ \\ Spectroscopy Division, ${ }^{+}$Centre for Design and Manufacture, Bhabha Atomic \\ Research Centre, Mumbai 400 085, India \\ e-mail: dibyendu@magnum.barc.ernet.in
}

MS received 1 December 2003; revised 4 June 2004

\begin{abstract}
An extended X-ray absorption fine structure (EXAFS) beam-line for $\mathrm{X}$-ray absorption studies using energy dispersive geometry and position sensitive detector is being developed for the INDUS-II synchrotron source. The optical design of the beam-line has been completed based on the working principle that a single crystal bent in the shape of an ellipse by a crystal bender would act as a dispersing as well as focusing element. The heart of the beam-line is the crystal bender which has been designed on the basis of the principle of four-point bending and has been fabricated indigenously. The crystal bender is capable of producing pre-defined elliptical curvature on a crystal surface by applying different couples at the two-ends of the crystal which has variable width along its length. The focusing property of the crystal bender has been tested using a laser source and has been compared with the theoretically simulated results.
\end{abstract}

Keywords. Synchrotron; EXAFS; elliptical crystal bender.

\section{Introduction}

The INDUS-II synchrotron source is a $2.5 \mathrm{GeV}$ source which is presently being commissioned at the Centre for Advanced Technology, Indore (http://www.cat.ernet.in). An extended Xray absorption fine structure (EXAFS) beam-line for X-ray absorption studies, using energy dispersive geometry and position sensitive detector, has been proposed to be installed at a bending magnet port of the INDUS-II Synchrotron source (Das et al 1999).

The EXAFS technique deals with measurements of fine structures in the X-ray absorption spectra above the absorption edge of different species of atoms in a material (Sayers et al 1971). With the advent of the modern bright synchrotron radiation sources, EXAFS measurement has emerged out to be the most powerful structure determination technique that can be applied to any type of material, e.g., amorphous solids, liquids, solution, gases, polymers and surfaces (Lytle 1999; Stern 2001; Tournus et al 2002; Hricovini et al 2003; Ascone et al 2003). The

${ }^{*}$ For correspondence 


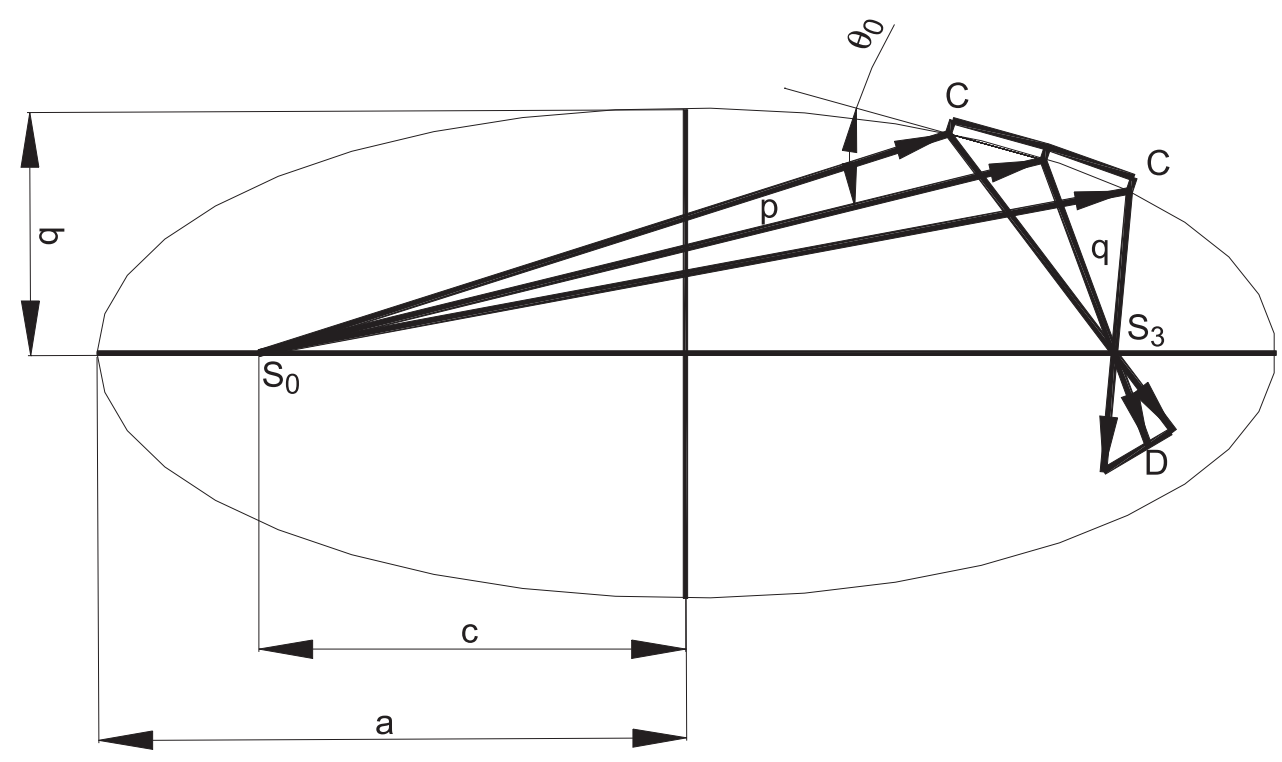

Figure 1. Schematic diagram showing the principle of action of the beam-line.

proposed EXAFS beam-line at INDUS-II will carry out the X-ray absorption measurements in energy dispersive mode involving no time-consuming scanning mechanism and thus can be applied to study in-situ fast and time-resolved processes (Iwasawa 2003).

A schematic diagram describing the principle of action of the beam-line is shown in figure 1. The basic idea is to use a single crystal (CC) bent in the shape of an ellipse in a particular fashion such that the source $\left(S_{0}\right)$ and the sample positions $\left(S_{3}\right)$ are situated at the two foci of the ellipse (Lee et al 1994). White synchrotron radiation from the source $\left(S_{0}\right)$ will be made incident on the crystal and depending on the angle of incidence of the beam and its radius of curvature, the crystal will reflect a particular central energy $\left(E_{o}\right)$ with a certain bandwidth $(\Delta E)$ and this spatially dispersed polychromatic radiation will then be focused at the sample position $\left(S_{3}\right)$. The transmitted radiation from the sample will then be detected by a position-sensitive detector (D). Thus the energy dispersed absorption spectra of the sample over the whole band width $(\Delta E)$ around the central energy $\left(E_{o}\right)$ will be recorded on the detector simultaneously. The proposed beam-line will cover the photon energy range of $5 \mathrm{keV}$ to $20 \mathrm{keV}$ by a bent $\mathrm{Si}(111)$ crystal having $2 d$ value equal to $6 \cdot 2709 \AA$. The beam-line will provide band widths of the order of $0.3 \mathrm{keV}, 1 \mathrm{keV}$ and $2 \mathrm{keV}$ at photon energies of $5 \mathrm{keV}$, $10 \mathrm{keV}$ and $20 \mathrm{keV}$ respectively. The average resolution at the detector has been estimated to be $\sim 1 \mathrm{eV}$ per channel (Das et al 1999).

The heart of the above beam-line is the crystal bender which would be used to generate the required elliptical curvature on the surface of the $\mathrm{Si}(111)$ crystal. Generally two types of mechanisms are used for bending of crystal to achieve sagittal (horizontal) focusing of the beam in Synchrotron beam-line optics. One method is to push the apex of an isosceles triangleshaped crystal while keeping its base fixed (Batterman \& Berman 1983; Flank et al 1983; Tolentino et al 1990). A perfect triangular crystal would give rise to a cylindrical curvature by this process while an elliptical shape can be approached by using a complicated profiled crystal. However, the more widely-used technique is the four-point bending mechanism where a rectangular crystal is held by two fixed inner rods and are pushed by two external moving 
rods (Sparks et al 1982; Lee et al 1994). The advantage of such a mechanism is that the part between the inner rods would have the exactly desired curvature and it prevents crystal twisting in the other plane which is detrimental to Bragg reflection (Stephens et al 1993; Artemev et al 2001). An elliptical curvature on the surface of a crystal can be exactly achieved by this process.

In the present communication, the design, development and testing of an indigenous crystal bender has been described, which has been developed based on the four-point bending principle discussed above and will be used for sagittal focusing of the synchrotron beam in the EXAFS beam-line at INDUS-II.

\section{Optical design of the beam-line}

The detailed optical layout of the beam-line is shown in figure 2 . The beam coming from the synchrotron source is first collimated by two copper blocks $(\mathrm{K}, \mathrm{K})$ kept at an angle of $15^{\circ}$ with the beam path. The collimated beam with horizontal divergence of $1.5 \mathrm{mrad}$ then falls on a Be window (B) of suitable thickness which help to cut-off the unwanted low-energy part from the continuum. The transmitted beam through the Be window falls on the Ta slit $\left(S_{1}\right)$ which defines the final divergence of the synchrotron beam. The beam emerging from slit with required vertical and horizontal divergences falls on the crystal (CC). After reflection from the crystal the dispersed beam passes through the second slit $\left(S_{2}\right)$. The slit $\left(S_{2}\right)$ helps in shielding the scattered radiation so that the beam gets sharply focused on the sample $\left(S_{3}\right)$. From the sample, the beam diverges further and finally gets detected at the CCD-type position sensitive detector (D). A plane mirror (M) is then used before the detector to cut-off the higher harmonics from the radiation diffracted by the Si crystal. W is the shielding wall that isolates the front-end of the beam-line from the rest portion.

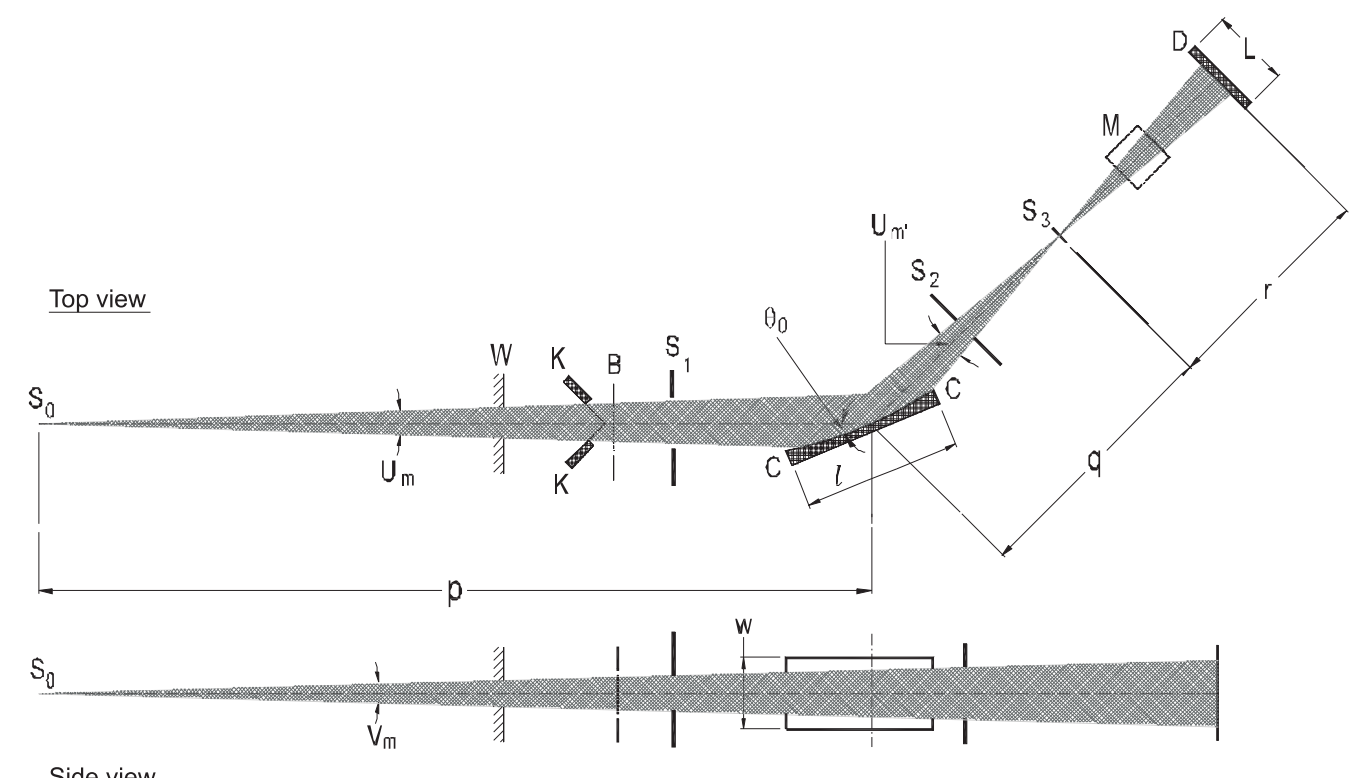

Side view

Figure 2. Optical layout of the beam-line. 
Table 1. EXAFS beam-line fixed parameters.

\begin{tabular}{lc}
\hline Parameter & Value \\
\hline Crystal type & $\operatorname{Si}(111)$ \\
$2 d$ value & $6 \cdot 2709 \AA$ \\
Source to crystal distance $(p)$ & $20,000 \mathrm{~mm}$ \\
Horizontal beam divergence $\left(U_{m}\right)$ & $1.5 \mathrm{mrad}$ \\
\hline
\end{tabular}

Some of the parameters of the beam-line which have been decided based on practical considerations, are listed in table 1 . An Si single crystal with (111) orientation $(2 d=6 \cdot 2709 \AA)$ has been chosen as the polychromator since it has a very narrow Darwin width, low thermal expansion coefficient and high thermal conductivity. The source to Si crystal distance $(p)$ has been fixed at $20,000 \mathrm{~mm}$ on practical considerations. A reasonable detector size of $25 \times 25 \mathrm{~mm}$ has been chosen considering the resolution, cost and availability of the CCD type position-sensitive detectors and horizontal beam divergence $\left(U_{m}\right)$ has been chosen to be 1.5 mrad taking into considerations the detector and crystal sizes and heat flux falling on the different components of the beam-line.

The parameters of the beam-line which are functions of the central energy of interest $\left(E_{o}\right)$ are shown in table 2 . The energy band widths $(\Delta E)$ at different central energies have been estimated considering practical values of the radius of curvature $(R)$ of the crystal. The values of the different other parameters shown in table 2 are determined as follows.

The Bragg angle $\theta_{0}$ for the central ray at the pole of the crystal is obtained from the central photon energy $E_{o}$ or wavelength $\lambda_{0}$ from the well-known Bragg relation (James 1962):

$$
\sin \theta_{o}=\lambda_{o} / 2 d=12 \cdot 398 / 2 d E_{o}(\mathrm{keV}) .
$$

Since the source to crystal distance $(p)$ and horizontal divergence $\left(U_{m}\right)$ are fixed, the length of the illuminated portion of the crystal $(l)$ corresponding to the central energy $E_{o}$ is then determined by the simple geometrical relation:

$$
U_{m}=(l / p) \sin \theta_{o} .
$$

For simplicity in calculations, initially we have assumed the elliptical crystal to a spherical cylinder having a uniform radius of curvature $\left(R_{0}\right)$. Since $l$ is known, the radius of curvature $\left(R_{0}\right)$ can be determined by the following relation for pre-specified value of the energy band width $(\Delta E)$ for all values of $E_{o}$ (Lemonmer et al 1978):

$$
\Delta E=E_{o} l \cot \theta_{o}\left[\left(1 / R_{0}-\sin \theta_{o} / p\right)\right]
$$

\begin{tabular}{|c|c|c|c|}
\hline \multirow{2}{*}{$\begin{array}{l}\text { Parameters } \\
\text { Photon energy }\left(E_{o}\right)\end{array}$} & \multicolumn{3}{|c|}{ Values } \\
\hline & $5000 \mathrm{eV}$ & $10,000 \mathrm{eV}$ & $20,000 \mathrm{eV}$ \\
\hline Band pass $(\Delta E)$ & $297 \mathrm{eV}$ & $1123 \mathrm{eV}$ & $2000 \mathrm{eV}$ \\
\hline Bragg angle $\left(\theta_{0}\right)$ & $23 \cdot 28^{\circ}$ & $11.40^{\circ}$ & $5 \cdot 67^{\circ}$ \\
\hline Crystal length $(l)$ & $75.9 \mathrm{~mm}$ & $151.8 \mathrm{~mm}$ & $303.5 \mathrm{~mm}$ \\
\hline Crystal radius $\left(R_{0}\right)$ & $2803 \mathrm{~mm}$ & $6287 \mathrm{~mm}$ & $26,550 \mathrm{~mm}$ \\
\hline Crystal to sample distance $(q)$ & $570 \mathrm{~mm}$ & $641 \mathrm{~mm}$ & $1404 \mathrm{~mm}$ \\
\hline
\end{tabular}

Table 2. EXAFS beam-line variable parameters. 
Table 3. Parameters of the elliptical cylinder shaped crystal.

\begin{tabular}{rrrrrrrr}
\hline$E_{o}(\mathrm{eV})$ & $p(\mathrm{~mm})$ & $q(\mathrm{~mm})$ & $\theta_{0}(\mathrm{deg})$ & $\alpha(\mathrm{deg})$ & $\mathrm{a}(\mathrm{mm})$ & $\mathrm{b}(\mathrm{mm})$ & $\mathrm{c}(\mathrm{mm})$ \\
\hline 5,000 & 20,000 & 570 & $23 \cdot 28$ & $66 \cdot 72$ & 10285 & $1335 \cdot 5$ & 10198 \\
10,000 & 20,000 & 641 & 11.40 & $78 \cdot 60$ & 10320 & $708 \cdot 1$ & 10296 \\
20,000 & 20,000 & 1404 & $5 \cdot 67$ & 84.33 & 10702 & 524 & 10689 \\
\hline
\end{tabular}

Since the values of $p, \theta_{0}$ and $R_{0}$ are known, the crystal to sample distance $(q)$ is then determined from (4) given below (Lemonmer et al 1978):

$$
1 / \sin \theta_{o}=R_{0} / 2[(1 / p+1 / q)] .
$$

The above calculations have been done for various photon energies and the computed data corresponding to two extreme photon energies of $5 \mathrm{keV}$ and $20 \mathrm{keV}$ and at an intermediate photon energy of $10 \mathrm{keV}$ are shown in table 2 . The data presented in table 1 and table 2 have been used in the ray-tracing programme "SHADOW" (SHADOW GUI 1997) to check the focusing of the diffracted beam at the sample position and the available resolution at the detector position. The detailed results of this exercise have been reported elsewhere (Das et al 1999, 2002, 2003). The above calculations have been done first assuming the crystal to be bent in the shape of a spherical cylinder. However, as has been discussed earlier, for perfectly focussing the polychromatic beam at the sample position, the crystal has to be bent in the shape of an elliptical cylinder as shown in figure 1. The ray-tracing work has also been carried out assuming an elliptically bent crystal and the geometrical parameters of the ellipse, viz., the semi-major (a) and semi-minor (b) axes, as defined in figure 1, have been obtained by the ray-tracing analysis using the values of $\theta_{0}, p$ and $q$ for different central photon energies $\left(E_{o}\right)$. The parameters of the ellipse thus obtained are shown in the table 3 for three different photon energies of $5 \mathrm{keV}, 10 \mathrm{keV}$ and $20 \mathrm{keV}$. Design of the crystal bender has been made in such a way that the above elliptical parameters can be generated on the surface of the crystal at different Bragg angles.

\section{Mechanical design of the crystal bender}

As has been mentioned earlier, the crystal bender that has been described in the present communication, works on the principle of four-point bending. The schematic mechanical design of this bender is shown in figure 3. In this bender the crystal is held in proper position, pivoted at two points $(\mathrm{P}, \mathrm{P})$ and is pushed from the back at two other points $(\mathrm{Q}, \mathrm{Q})$ by a wormshaft and worm-wheel arrangement. The mechanism gives rise to two couples at the two ends of the crystal. The worm wheels are driven by stepper motors to produce precision torques on the crystal.

For a crystal of length $L$ and applied couples $C_{1}$ and $C_{2}$ at two ends, the bending of the crystal is given by the following Bernouilli-Euler equation (Padmore 1996):

$$
\begin{aligned}
E I \frac{\mathrm{d}^{2} y}{\mathrm{~d} x^{2}} & =\frac{C_{1}+C_{2}}{2}-\frac{C_{1}-C_{2}}{L} x, \\
I & =b h^{3} / 12,
\end{aligned}
$$




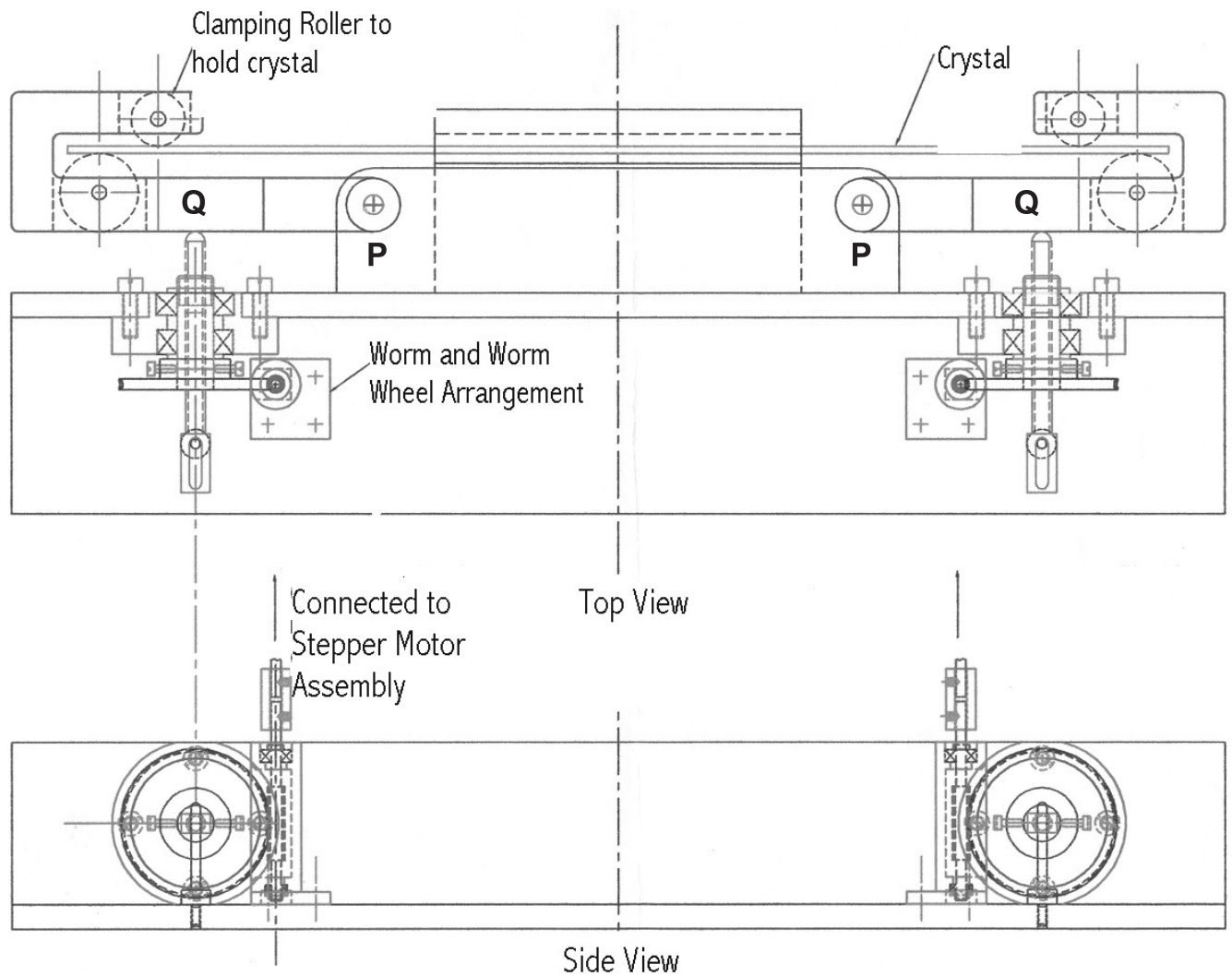

Figure 3. Mechanical design of the crystal bender.

where $y$ is the displacement at any point $x$ along the length of the crystal, $E$ is Young's modulus, $I$ is the section modulus or moment of inertia, $b$ is the mechanical width of the crystal, and $h$ is the thickness of the crystal.

The second derivative in (5) is the curvature of the ellipse and it is a function of the distance $(x)$ of any point along the crystal from its centre $(x=0)$. Since $C_{1}$ and $C_{2}$ are independent of $x$, the quantity $I$ has to be varied, either by changing the width $b$ or thickness $h$ to get the required elliptical shape of the bent crystal. In the present arrangement the crystal width $b$ has been chosen to vary. The length of the crystal $(L)$ has been estimated to be $410 \mathrm{~mm}$ and its thickness $(h)$ and width at $x=0\left(b_{0}\right)$ have been taken to be $3 \mathrm{~mm}$ and $30 \mathrm{~mm}$ respectively.

Assuming first that the crystal is bent in the shape of a spherical cylinder with constant radius of curvature $R_{o}$, the values of the two couples can be found out from (5) as (Padmore 1996):

$$
\begin{aligned}
& C_{1}+C_{2}=2 E I_{o} / R_{o}, \\
& C_{1}-C_{2}=\left(3 E I_{o} L \sin \alpha / 2 R_{o}\right)[(1 / p)-(1 / q)],
\end{aligned}
$$

where $R_{0}$ is the radius of curvature, $I_{0}$ is the value of section modulus at $x=0$, and $\alpha\left(=90-\theta_{0}\right)$ is the angle of incidence while the parameters $p$ and $q$ have been defined earlier in figure 1 as well as in tables 1 and 2. 
Now, in our case (Ygural and Senster 1995),

$$
\begin{aligned}
& E=7000 \mathrm{~kg} / \mathrm{mm}^{2} \text { (for Si) and } \\
& I_{0}\left(=b_{0} h^{3} / 12\right)=67.5 \mathrm{~mm}^{4} .
\end{aligned}
$$

For a mean energy (say of order $10 \mathrm{keV}$ ), as shown in table 2, radius of curvature at $x=0$, i.e., $R_{0}=6287 \mathrm{~mm}$.

Substituting all values, in (7 and 8$)$ we have

$$
\begin{aligned}
& C_{1}+C_{2}=150 \cdot 66, \\
& C_{1}-C_{2}=-68 \cdot 58 .
\end{aligned}
$$

Solving these two equations, we get

$$
C_{1}=41.03 \mathrm{~kg} \mathrm{~mm}, C_{2}=109.62 \mathrm{~kg} \mathrm{~mm}
$$

Since for an ellipse, radius of curvature varies at various points, the elliptical surface required corresponding to a certain energy (given in table 3 ) has been divided into a number of small segments so that each segment remains small enough to assume constant radius of curvature within that segment. Normals are drawn on the tangents at the two end sections of each segment and the intersection of these two normals gives the centre of curvature of that segment. The radial distance from this centre of curvature to the mid point of the segment gives the radius of curvature for that segment. Radius of curvature of each segment corresponding to the mean energy of $10 \mathrm{keV}$ is given in table 4, where the $410 \mathrm{~mm}$ long crystal has been divided into several small segments, each of length $5 \mathrm{~mm}$.

Putting in the values of $C_{1}$ and $C_{2}$ in Bernouilli's equation (5) and considering that

$$
\mathrm{d}^{2} y / \mathrm{d} x^{2}=1 / R(x), \text { we have } b(x) / R(x)=0 \cdot 00478285+0 \cdot 00001062 x .
$$

In the above equation, substituting for $x$ and the corresponding $R(x)$ which has already been determined, variation in width $b(x)$ of the crystal is calculated. The $b(x)$ values for the $10 \mathrm{keV}$ energy are also shown in table 4 . The same calculations have been done for various other energies also and the $b(x)$ values have been determined. The typical shape of the Si crystal required for $10 \mathrm{keV}$ energy is shown in figure 4 . Taking into considerations the mechanical requirement of clamping the total crystal length has been taken to be $460 \mathrm{~mm}$.

Software simulation of crystal bending has been done by finite element modelling and analysis using SDRC's I-DEAS Master Service Version-8 code. A finite element model of the $\mathrm{Si}$ crystal between the two points $(\mathrm{Q}, \mathrm{Q})$, as shown in figure 3, is created using thin shell elements of $3 \mathrm{~mm}$ thickness. Physical properties in the form of thickness and material properties such as modulus of elasticity and Poission's ratio are supplied from the literature (Ygural \& Senster 1995). To simulate the actual bending with application of couples, the appropriate boundary condition is applied. The nodes at the two edges are allowed to rotate about their own axes which are parallel to the plane of the crystal. To arrest the rigid body mode, movements of all nodes at the left edge are restrained in the $X, Y$ and $Z$ directions. The co-ordinate system convention assumed here is shown in figure 5 . As the bending occurs, the right edge of the crystal moves towards the left edge, hence all the nodes of the right edge are allowed to move in the $X$-direction only. The results obtained for the deflection of crystal corresponding to $10 \mathrm{keV}$ photon energy is shown in figure 5 . Values of deflection at each node along the length of the crystal have been found and compared with the actual 
Table 4. Radius of curvature and variation of width of crystal along length for $10 \mathrm{keV}$ energy.

\begin{tabular}{|c|c|c|c|c|c|}
\hline$x(\mathrm{~mm})$ & $R(x)(\mathrm{mm})$ & $b(x)(\mathrm{mm})$ & $x(\mathrm{~mm})$ & $R(x)(\mathrm{mm})$ & $b(x)(\mathrm{mm})$ \\
\hline-205 & 9310 & $24 \cdot 260$ & 5 & 6202 & 29.992 \\
\hline-200 & 9233 & 24.549 & 10 & 6133 & 29.984 \\
\hline-195 & 9155 & $24 \cdot 827$ & 15 & 6063 & 29.964 \\
\hline-190 & 9077 & 25.098 & 20 & 5995 & 29.946 \\
\hline-185 & 9000 & $25 \cdot 363$ & 25 & 5926 & 29.916 \\
\hline-180 & 8927 & $25 \cdot 617$ & 30 & 5857 & $29 \cdot 879$ \\
\hline-175 & 8845 & $25 \cdot 865$ & 35 & 5789 & 29.839 \\
\hline-170 & 8768 & $26 \cdot 106$ & 40 & 5721 & 29.792 \\
\hline-165 & 8691 & $26 \cdot 338$ & 45 & 5654 & 29.744 \\
\hline-160 & 8615 & $26 \cdot 565$ & 50 & 5586 & 29.683 \\
\hline-155 & 8538 & $26 \cdot 781$ & 55 & 5519 & $29 \cdot 620$ \\
\hline-150 & 8462 & $26 \cdot 992$ & 60 & 5452 & $29 \cdot 550$ \\
\hline-145 & 8386 & $27 \cdot 195$ & 65 & 5386 & 29.478 \\
\hline-140 & 8310 & $27 \cdot 390$ & 70 & 5319 & $29 \cdot 394$ \\
\hline-135 & 8234 & 27.576 & 75 & 5253 & $29 \cdot 308$ \\
\hline-130 & 8159 & $27 \cdot 758$ & 80 & 5187 & $29 \cdot 215$ \\
\hline-125 & 8084 & 27.933 & 85 & 5121 & $29 \cdot 115$ \\
\hline-120 & 8009 & 28.099 & 90 & 5056 & $29 \cdot 014$ \\
\hline-115 & 7934 & $28 \cdot 257$ & 95 & 4991 & 28.906 \\
\hline-110 & 7859 & 28.407 & 100 & 4926 & 28.791 \\
\hline-105 & 7789 & $28 \cdot 553$ & 105 & 4861 & 28.669 \\
\hline-100 & 7711 & 28.691 & 110 & 4797 & 28.547 \\
\hline-95 & 7637 & 28.821 & 115 & 4733 & $28 \cdot 417$ \\
\hline-90 & 7563 & 28.943 & 120 & 4669 & $28 \cdot 281$ \\
\hline-85 & 7489 & $29 \cdot 058$ & 125 & 4606 & $28 \cdot 144$ \\
\hline-80 & 7416 & $29 \cdot 168$ & 130 & 4542 & 27.994 \\
\hline-75 & 7343 & $29 \cdot 271$ & 135 & 4479 & $27 \cdot 843$ \\
\hline-70 & 7270 & $29 \cdot 366$ & 140 & 4417 & 27.693 \\
\hline-65 & 7197 & 29.454 & 145 & 4354 & 27.529 \\
\hline-60 & 7124 & $29 \cdot 533$ & 150 & 4292 & $27 \cdot 365$ \\
\hline-55 & 7052 & $29 \cdot 609$ & 155 & 4230 & $27 \cdot 194$ \\
\hline-50 & 6980 & $29 \cdot 677$ & 160 & 4169 & $27 \cdot 023$ \\
\hline-45 & 6908 & $29 \cdot 738$ & 165 & 4107 & $26 \cdot 839$ \\
\hline-40 & 6836 & 29.791 & 170 & 4046 & $26 \cdot 656$ \\
\hline-35 & 6765 & $29 \cdot 841$ & 175 & 3986 & $26 \cdot 472$ \\
\hline-30 & 6694 & $29 \cdot 883$ & 180 & 3925 & $26 \cdot 275$ \\
\hline-25 & 6623 & 29.918 & 185 & 3865 & $26 \cdot 079$ \\
\hline-20 & 6552 & 29.945 & 190 & 3805 & $25 \cdot 876$ \\
\hline-15 & 6482 & 29.969 & 195 & 3746 & $25 \cdot 674$ \\
\hline-10 & 6411 & 29.982 & 200 & 3687 & $25 \cdot 465$ \\
\hline-5 & 6341 & 29.994 & 205 & 3626 & $25 \cdot 235$ \\
\hline 0 & 6272 & $30 \cdot 000$ & & & \\
\hline
\end{tabular}




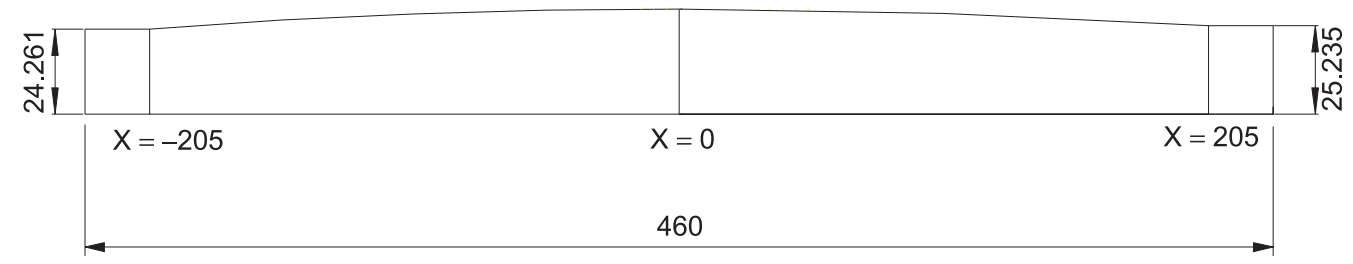

Figure 4. Shape of the crystal required to achieve the elliptic bending for $10 \mathrm{keV}$ photon energy.

deflection required to achieve the elliptical shape. The results are found to agree well within acceptable limits. Figure 6 shows the stress distribution in the crystal in the bent condition corresponding to $10 \mathrm{keV}$ photon energy. Maximum principal stress induced in the crystal is found to be $0.758 \mathrm{kgf} \mathrm{mm}^{-2}$ which is less than the maximum allowable stress.

\section{Testing of the crystal bender}

Figure 7 shows the photograph of the crystal bender fabricated indigenously based on the above design. Two PC-controlled stepper motors are used to push the warm shafts for providing the required torques at the two ends of the crystals. The crystal bender has been tested both mechanically and optically. The set-up for optical testing of the crystal bender is shown

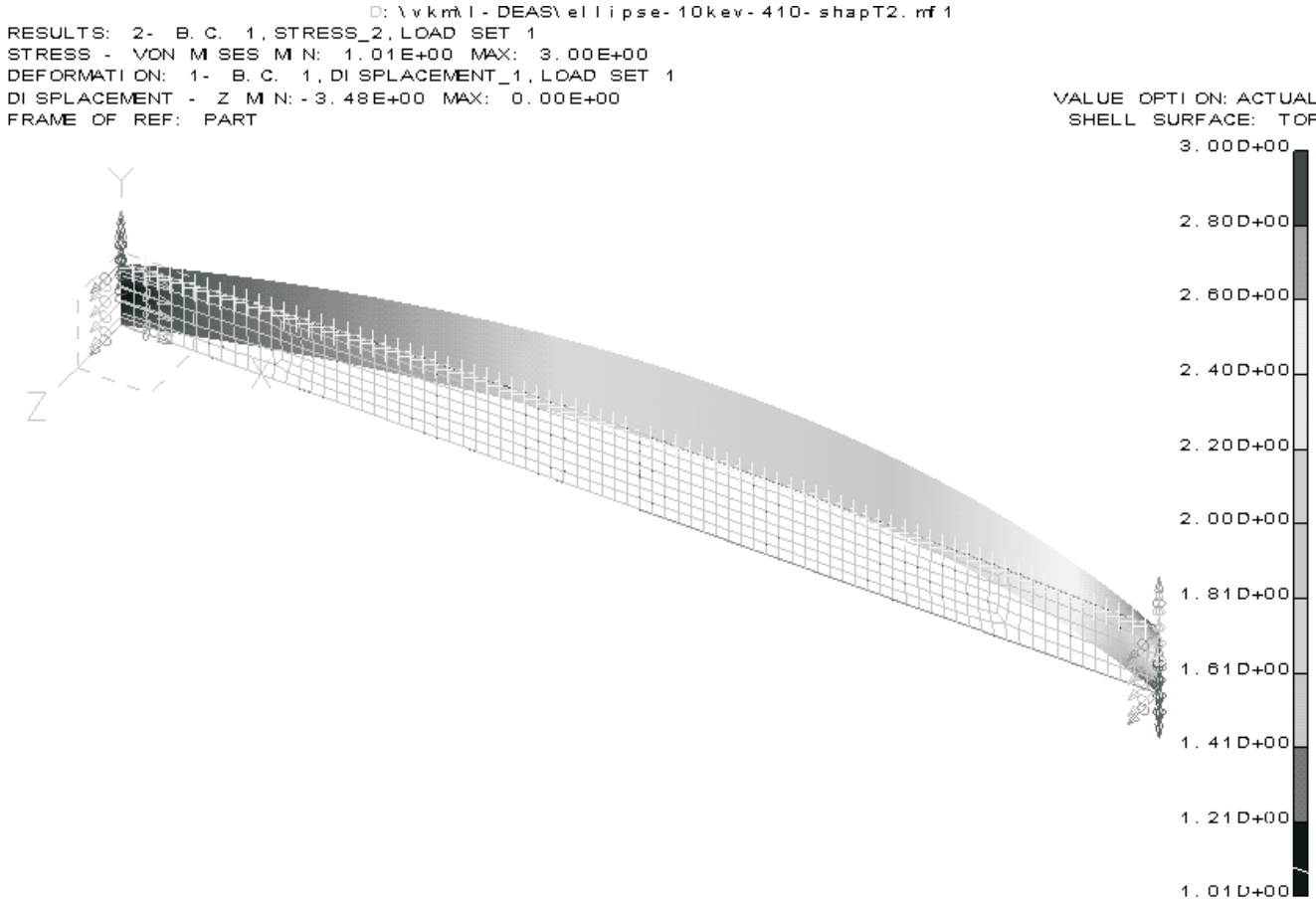

Figure 5. Deflection analysis of crystal ( $\mathrm{mm})$ for bending profile required for $10 \mathrm{keV}$ photon energy. (The values of the deflection in $\mathrm{mm}$ are represented by the gray codes shown in the right-most portion of the diagram.) 


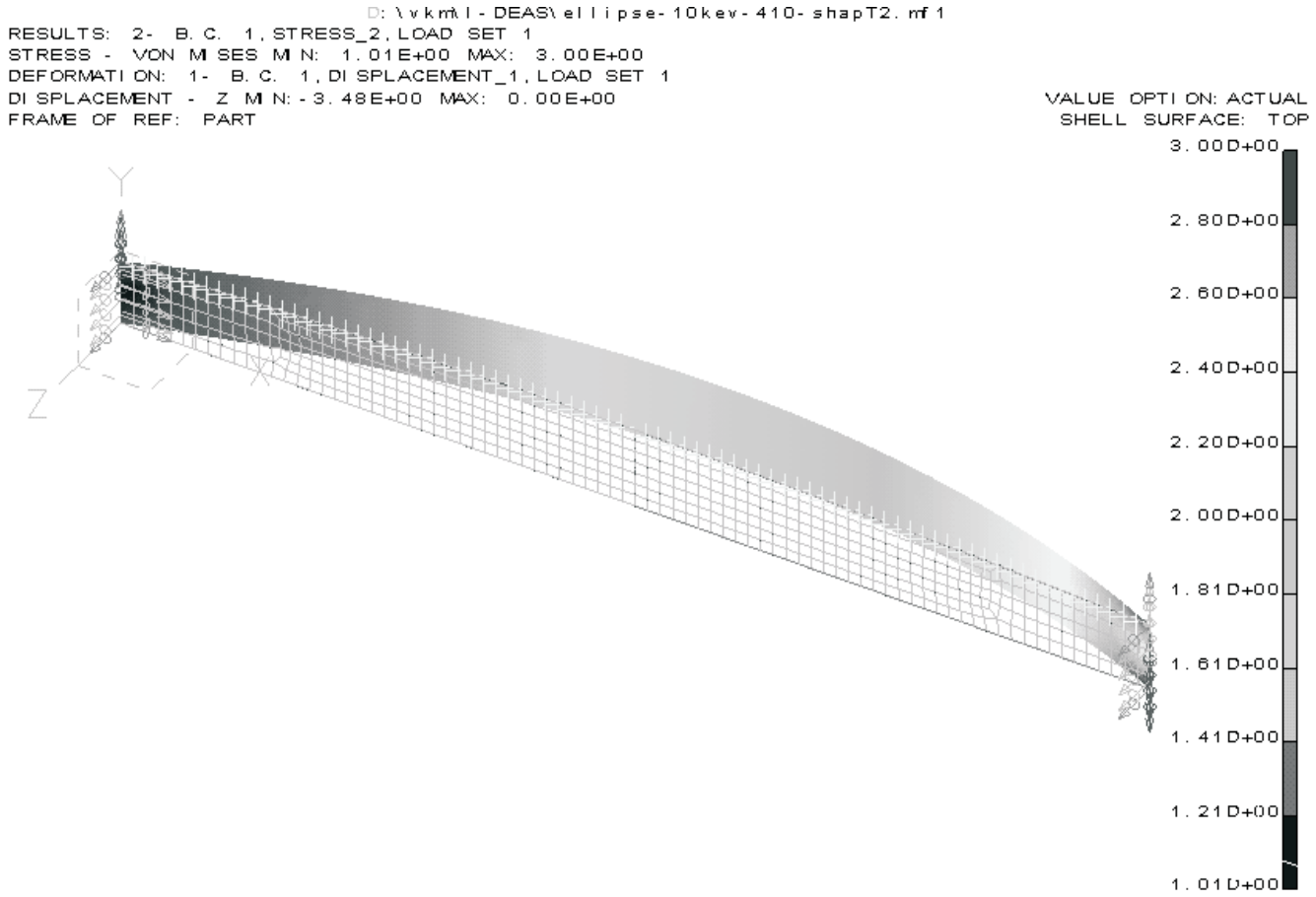

Figure 6. Stress analysis of crystal $\left(\mathrm{kgf} \mathrm{mm}^{-2}\right)$ for the bending profile required for $10 \mathrm{keV}$ photon energy. (The values of the stress generated in the different portion of the crystal in $\mathrm{kgf} \mathrm{mm}^{-2}$, are represented by the gray codes shown in the right-most portion of the diagram.)

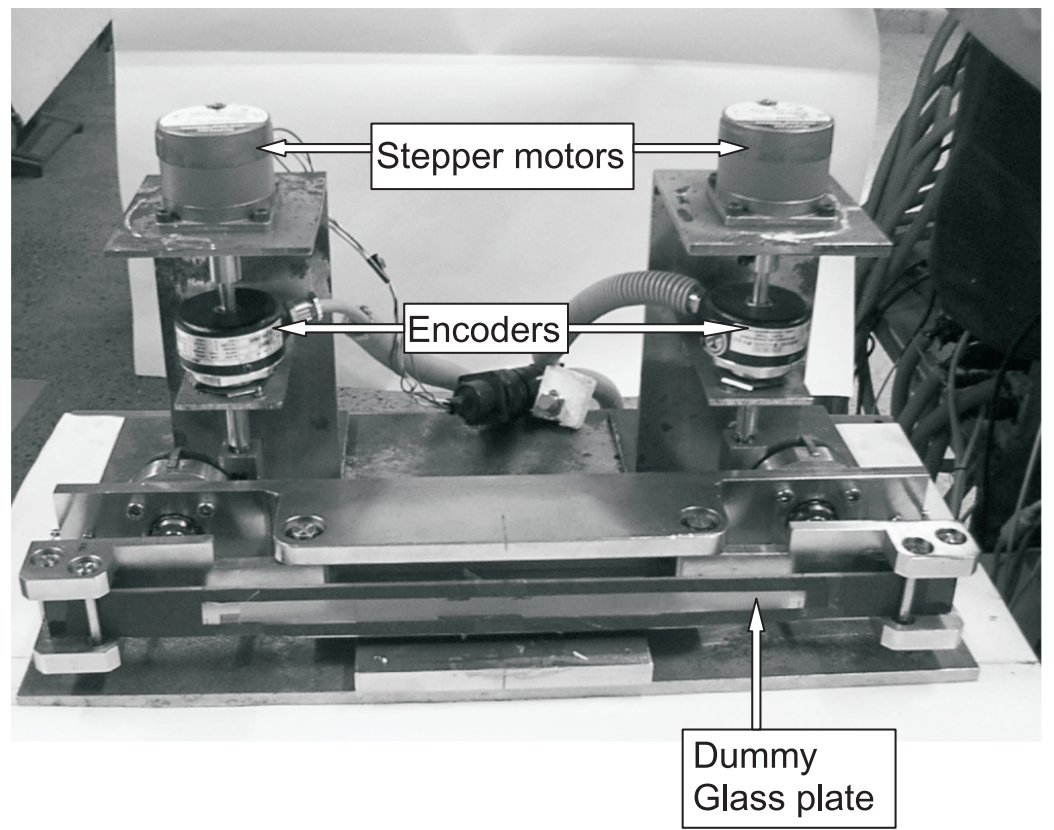

Figure 7. Photograph of the indigenously developed crystal bender. 


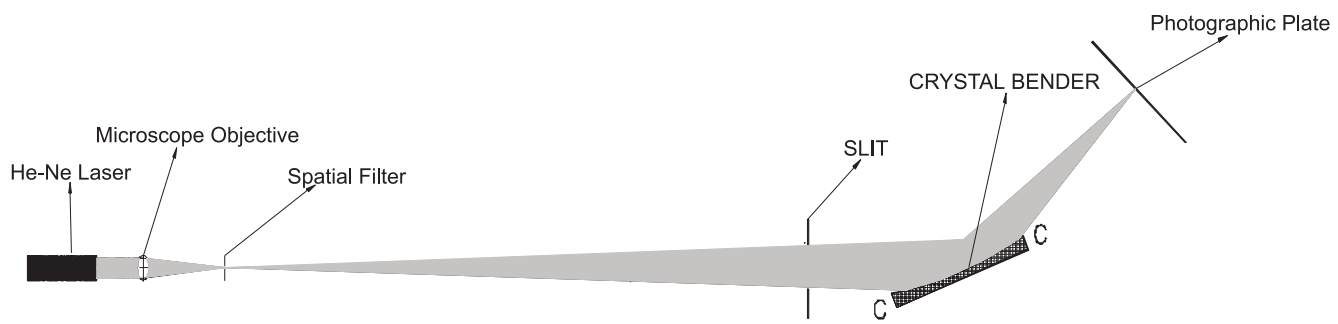

Figure 8. Schematic diagram showing the arrangement for testing of crystal bender.

schematically in figure 8. Light from an He-Ne laser source has been focussed by a microscope objective on the pin hole of a spatial filter to simulate a synchrotron source. The light diverging from the spatial filter is then made incident upon the crystal mounted on the crystal bender which is kept at a distance of $20 \mathrm{~m}$ from the source. The crystal is elliptically bent to different radius of curvatures using different torques at the two ends of the crystal bender and the focus spot has been monitored on a ground glass plate as a function of grazing angle of incidence and the mean radius of curvature of the ellipse. It has been observed that the width of the best focus spot obtained is $300 \mu \mathrm{m}$ at a distance of $641 \mathrm{~mm}$ from the centre of the crystal and at an grazing angle of incidence of $11.40^{\circ}$. This is equivalent to the configuration for Bragg reflection of $10 \mathrm{keV}$ as can be found from table 2. Figure 9 shows the photograph of

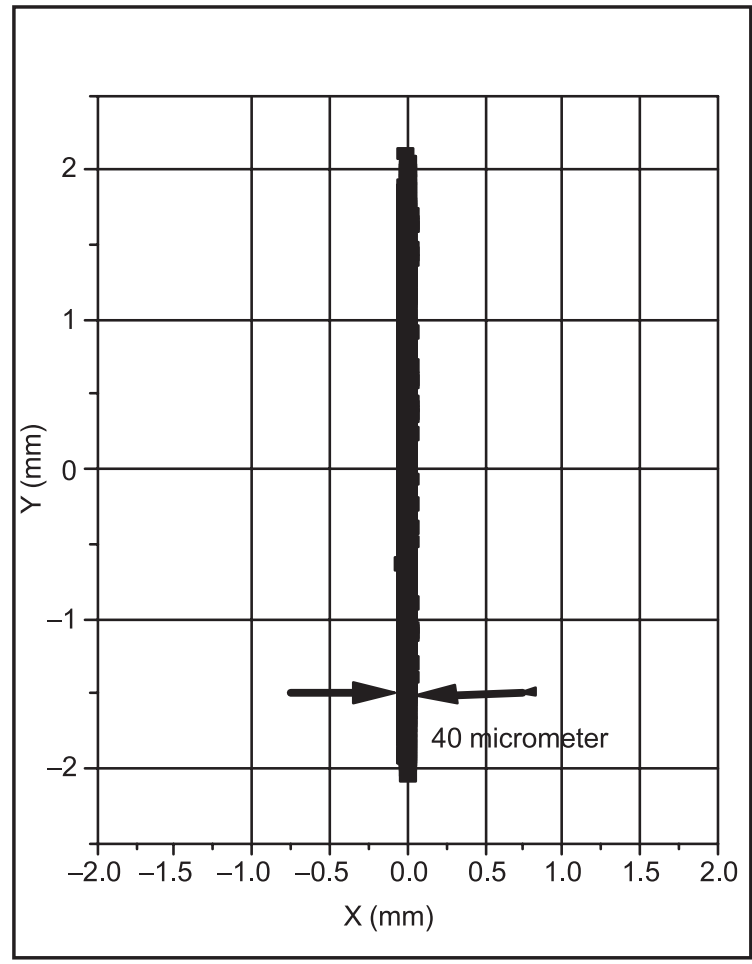

(a)
Elliptically Bent Si (111) Crystal

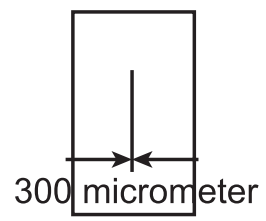

Grazing Angle of Incidence: $11.40 \mathrm{deg}$ Source Distance: Image Distance:

(b)

Figure 9. Image spot at the focus position of the crystal: (a) Theoretical; (b) experimental. 
the focus spot at the above condition along with the focus spot simulated theoretically (using the ray-tracing programme "SHADOW") under the same condition with a synchrotron beam. The difference between the widths of the simulated and experimental focal spots arises due to the fact that the latter spot is formed by specular reflection of the optical beam from a real surface while the former is generated assuming Bragg reflection of X-rays from crystal planes.

\section{Conclusion}

A crystal bender has been designed and fabricated indigenously for using in a dispersive EXAFS beam-line to be set up at INDUS-II synchrotron source. The bender will be used to bend $\operatorname{Si}(111)$ crystals in elliptical shape for sagittal focusing of the synchrotron beam on the sample. The function of the bender has been tested optically using a divergent laser beam. The focus spots obtained at the sample position were recorded on photographic plate and have been compared with the theoretically generated focus spots.

\section{References}

Ascone I, Meywr-Klaucke W, Murphy L 2003 Experimental aspects of biological X-ray absorption spectroscopy. J. Synchr. Rad. 10: 16-22

Artemev A, Artemiev N, Busetto E, Franc F, J Hrdy, Mraźek D, Savoia A 2001 Design, construction and tests of a crystal bender which provides constant position of the central part of the crystal. Nucl. Instrum. Methods Phys. Res. A467-468: 377-379

Batterman B W, Berman L 1983 Sagittal focusing of synchrotron radiation. Nuclear Instrum. Methods 208: $327-331$

Das N C, Jha S N, Roy A P 1999 Optical design of an X-ray absorption spectroscopy beam-line at INDUS-2 synchrotron radiation source. Report No. E/035 Bhabha Atomic Research Centre, Mumbai

Das N C, Jha S N, Bhattacharyya D, Sinha A K, Mishra V K, Verma Vishnnu, Ghosh A K 2002 Optical and mechanical design of the extended X-ray absorption fine structure (EXAFS) beam-line at INDUS-II synchrotron source. Report No. E/030, Bhabha Atomic Research Centre, Mumbai

Das N C, Jha S N, Bhattacharyya D 2003 Design of the extended X-ray absorption fine structure (EXAFS) beam-line at INDUS-II synchrotron source. Presented at the 8th Int. Conf. Synchrotron Radiation Instrumentation, San Fransisco, CA, USA

Flank A M, Fontaine A, Jucha A, Lemonnier M, Raoux D, Williams C 1983 EXAFS in dispersive mode. Nuclear Instrum. Methods 208: 651-654

Hricovini K, Padova P De, Quaresima C, Perfetti P, Brochier R, Richter C, Ilakovac V, Heckmann O, Lechevallier L, Bencok P, Fevre P Le, Teodorescu C 2003 Atomic structure and magnetic properties of Mn on InAs(1 0 0). Appl. Surf. Sci. 212-213: 17-25 http://www.cat.ernet.in

Ice G E, Sparks C J 1988 A simple cantilevered mirror for focusing synchrotron radiation. Nucl. Instrum. Meth. Phys. Res. A266: 394-398

Iwasawa Y 2003 In situ characterization of supported metal catalysts and model surfaces by timeresolved and three-dimensional XAFS techniques. J. Catal. 216: 165-177

James R W 1962 The optical principle of the diffraction of X-rays (London: G Bell \& Sons)

Koch E E (ed.) 1983 Handbook of synchrotron radiation (New York: North Holland) vol. 1a, ch. 1

Lemonmer M, Fourme R, Rousseaux F, Kahn R 1978 X-ray curved crystal monochromator system at the storage ring DCI. Nucl. Instrum. Methods 152: 173-177

Lee P L, Beno M A, Jennings G, Ramanathan M, Knapp G S, Huang K, Bai J, Montano P A 1994 An energy dispersive X-ray absorption spectroscopy beam-line, X6A, at NSLS. Rev. Sci. Instrum. 65: $1-6$ 
Lytle F W 1999 The EXAFS family tree: a personal history of the development of extended X-ray absorption fine structure. J. Synchr. Rad. 6: 123-134

Padmore H A 1996 Soft X-ray optics for spectro-microscopy at the advanced light source. Presented at the 17th Int. Conf. X-ray and Inner-shell Processes, Hamburg, Germany

Sayers D E, Stern E A, Lytle F W 1971 New technique for investigating non-crystalline structures: Fourier analysis of the extended X-ray absorption fine structure. Phys. Rev. Lett. 27: 1204-1207

SHADOW GUI 1997 Version 1.1.0 http://www.xraylith.wisc.edu/shadow/GUI

Sparks C J, Ice G E, Wong J, Batterman B W 1982 Sagittal focusing of synchrotron X-radiation with curved crystal. Nuclear Innstum. Methods 194: 73-78

Stephens P W, Eng P J, Tse T 1993 Construction and performance of a bent crystal monochromator. Rev. Sci. Instrum. 64: 374-378

Stern E A 2001 Musings about the development of XAFS. J. Synchr. Rad. 8: 49-54

Tolentino H, Baudelet F, Dartyge E, Fontaine A, Lena A, Tourillon G 1990 Aberration-free and harmonic-free optics for time-resolved X-ray absorption spectroscopy using synchrotron radiation. Nucl. Instrum. Meth. Phys. Res. A289: 307-316

Tournus F, Masenelli B, Melinon P, Blasé X, Perez A, Pellkarin M, Broyer M, Flank A M, Lagarde P 2002 Bridging $\mathrm{C}_{60}$ by silicon: Towards non-Van der Waals $\mathrm{C}_{60}$-based materials. Phys. Rev. B65: $165417-165422$

Ygural A C, Senster S K 1995 Advanced strength and applied elasticity (Englewood Cliffs, NJ: Prentice-Hall) 